\title{
Magnetic resonance imaging and spectroscopy of the prostate
}

\author{
Carolyn Mountford
}

Published online: 20 November 2008

(C) ESMRMB 2008

The goal of this special issue of MAGMA is to provide an insight into the current status of MR imaging and spectroscopy as applied to the diagnosis and management of prostatic disease. The issue contains articles on current clinical approaches and cutting edge research.

The development of the endorectal coil in 1986 by the Hospital of the University of Pennsylvania team paved the way for staging the local extent of prostate cancer by MRI. The method was embraced by the urology community and was quickly integrated into routine radiologic protocols. With a rebate in place there was good reason to expedite its usage, as the method was important to the pre-surgical planning. The accuracy of the MRI method is reader-dependent, and many hospitals as part of their quality control compare the reader with the subsequent clinical diagnosis. The criteria by which the MRI is read are reliant on the training and experience of the reader and will remain so for the immediate future until pattern-recognition methods are integrated into the MR scanners. In this special issue there are two reports on the current usage of the endorectal coil for MRI of the prostate in clinical practice.

The MRI contrast behavior of adenocarcinoma of the prostate, compared to surrounding healthy prostate tissue and benign prostatic hyperplasia, remains ambiguous in many cases. Several leading MR spectroscopy groups have shown that significant differences in the metabolic state of the different prostate pathologies are discernable by proton MR spectroscopy using both single-voxel and spectroscopic imaging. The combination of MRI with MR spectroscopy, as

C. Mountford $(\varangle)$

Department of Radiology, Center for Clinical Spectroscopy,

Brigham and Women's Hospital, Harvard Medical School,

1640 Tremont St, BC-3-010-CC, Boston, MA 02120, USA

e-mail: cemountford@bics.bwh.harvard.edu;

cmountford@partners.org for breast, looks to offer an important increase in accuracy. Unfortunately, to date, this remains site-dependent.

During the peer-review process for this issue, a serious frustration became apparent in the urological and surgical communities pertaining to the time taken to translate spectroscopy as a robust tool into the prostate clinic. One senior surgeon stated that he was "tired of reading exaggerated claims filled with false promises".

The premise that MR spectroscopy will provide an increase in diagnostic accuracy, when added to the standard MRI exam, has been supported by the small number of multidisciplinary sites that are adept at using the technology in a clinical setting. It is fair to conclude that the urological surgeons in those hospitals quickly became dependent on the preoperative diagnosis and local staging made available. Indeed, these surgeons regularly report their confidence in the technology at meetings.

The means by which MR spectroscopy of the prostate was introduced was clearly unsuccessful and was assessed by the American College of Radiology Imaging Network (ACRIN) trial (ACRIN 6659). Some hospitals, aware of the potential pitfalls ahead, declined to participate in this study. I was asked by some to shed light on what went so badly wrong. After visiting only four sites the first serious issues emerged. The two inexperienced sites had technologists who relied solely on the automated software on their MR scanner. Undertaking clinical spectroscopy requires either a specially trained technologist or a scientist to operate the scanner. Those sites that have high diagnostic accuracy are manned by interactive technologists of the highest caliber. Conversely, many large hospitals, particularly in the USA, rotate the technologists around all of their many scanners. Such hospitals should not even try to do spectroscopy until the manufacturers find a way of automating high-quality spectroscopy data acquisition. 
Once good MR spectroscopy data are acquired from the prostate, the reading of each case requires knowledge of more than distinguishing a malignant from a benign spectrum. It requires a trained eye to distinguish voxels that contain benign or inflammatory disease. It also requires the identification of a voxel that contains a small percentage of malignant disease in an area of predominantly non-malignant tissue. Different zones in the prostate contain different chemistry. Each zone needs to be considered independently when comparing the chemistry and how it relates to pathology. Where a voxel covers two zones the population of each needs consideration. The ACRIN trial was set up to study the peripheral zone but accrued an unusually large number of central zone cancers. This would have contributed to the lower accuracy than expected. The MR spectroscopic differences between tissue specimens taken from the various zones is covered in this issue, as are differences in changes associated with tumor grade.

A second and European-driven study "An International Multi-Centre Assessment of Prostate MR Spectroscopy" (IMAPS), which includes sites in the USA and China, has been completed but the results are yet to be announced. The primary objectives of this multicenter and multinational trial are to prove that proton MR spectroscopic imaging allows for both detecting and localizing prostate carcinoma in the two major anatomic areas of the prostate, i.e., the peripheral zone and the central gland.

Clearly there has been little, if any, serious expenditure worldwide on training MR technologists in appropriate procedures for prostate spectroscopy. In 2008 those best equipped to read clinical prostate data are the spectroscopists who know when the data are of sufficient quality to warrant a diagnosis and how to interpret the data. The radiology community needs to decide either to embrace this technology or to pass it on to the urologists, as they did cardiac MR to the cardiologists.

Who are the stakeholders in relation to accurate preoperative diagnosis of prostate disease? The tax-paying patients are the most important stakeholders. Reduction in pain following surgery and therapy is often cited as an important issue for prostate cancer sufferers. A greater effort by the prostate MR community and the urological surgeons might well offer a better outcome by improvement in pre-surgical planning. Such improvements require a concerted effort and some leadership from the prostate cancer community.

The "Indian perspective" described in this issue illustrates some subtle differences in the chemical makeup of the diseased prostate between men from different populations. Whether this is an epigenetic or genetic factor (or both) remains to be determined.

The unambiguous assignment of resonances, using in vivo two-dimensional MR spectroscopy, is now becoming clinically viable. Two-dimensional MR spectroscopy will allow comparisons to be made and ascertain whether a biological reason exists for disparities in outcomes between cultures. Also described herein are the results from the correlation of MR imaging and spectroscopy parameters with genetic over-expression profiles in prostate cancer, which offers a welcome and overdue link between MR and genetics whilst at the same time allowing direct spatial correlation.

The papers in this special issue each show that there is significant information in the MR data from the diseased prostate. How this vast amount of information is translated into improved clinical care will depend upon larger patient studies and a closer working relationship with the manufacturers of the MR hardware. Multidisciplinary education programs, such as those implemented for neurospectroscopy on an international basis by the team based at Huntington Medical Center in Pasadena, ESMRMB "Lectures on MR" workshops and ISMRM will greatly assist in cross-fertilization between disciplines. Combining the results presented in this issue into a pattern-recognition software would provide for a rigorous method for unambiguous diagnosis in the future.

The choice of watchful waiting would be an easier decision if the patient and physician were sure of the diagnostic accuracy. MR spectroscopy could offer this diagnostic accuracy now, but there is a long way to go before the technology is available at more than a handful of clinics worldwide. 